Short report

\title{
Haematocrit, hypertension and smoking in patients with transient ischaemic attacks and in age and sex matched controls
}

\author{
MJG HARRISON, S POLLOCK, D THOMAS, JOHN MARSHALL \\ From the Department of Neurological Studies, the Middlesex Hospital and the National Hospital, Queen \\ Square, London, $U K$
}

SUMMARY The blood pressure, smoking habit and haemotocrit of 154 patients with transient ischaemic attacks and 191 age- and sex-matched neurological controls were studied. Regression analysis revealed that the haematocrit value was related to both systolic and diastolic blood pressure, and to smoking. Smoking elevated the haematocrit by $1 \cdot 19 \pm 0.59$ in males and by $2 \cdot 18$ \pm 0.68 in females. When these associations were allowed for there was still evidence of a higher haematocrit in patients with transient ischaemic attacks (plus $1.44 \pm 0.56$ in males and $0.75 \pm$ 0.75 in females $p<0.02$ ). The role of an elevated haematocrit in the pathogenesis of cerebrovascular disease and its management are briefly discussed.

Epidemiological evidence, particularly that obtained in the Framingham Study has highlighted the role of hypertension as a risk factor for stroke.' The same study demonstrated that a high haemoglobin level, partly related to hypertension, but also partly independently, increased the risk of thrombotic cerebrovascular accidents. Smoking also increased the risk of such events, and is responsible for elevated haematocrit levels. ${ }^{2}$ The interrelationship of these three risk factors has therefore been studied in patients referred to one of us (JM) for the investigation of attacks of focal neurological deficit lasting less than 24 hours.

\section{Method}

The case notes of 154 patients and 191 age- and sexmatched controls were studied for evidence of the casual blood pressure reading and haematocrit at the time of hospital consultation. All the patients had had one or more episodes of transient focal neurological deficit. None had had a recent completed stroke though some had in the past. The control patients were consulting neurological clinics for diverse problems including cervical spondylosis, lumbar spondylosis and motorneurone disease. The smok-

Address for reprint requests: Dr MJG Harrison, The Middlesex Hospital Medical School, London WIN 8AA, UK.

Received 5 January 1982

Accepted 30 January 1982 ing habit of the patients was also extracted from the records.

\section{Results}

There were 109 male and 46 female patients with one or more transient ischaemic attacks aged between 40 and 79 years. There were 119 males and 72 females with neurological problems unrelated to cerebrovascular disease. The age and sex distribution of the two groups is set out in table 1. Combining the data from all age groups there was evidence of a significantly higher haematocrit in the patients of either sex though the difference was small in absolute terms $(45.8 \pm 3.7$ of $44.0 \pm 3.7 \mathrm{p}<0.001$ Student $t$ ). A haematocrit of 49 or more was recorded in 19 male patients with transient ischaemia attacks and in 10 controls (chi square 4.15 $p<0.005$ ). Hypertension, arbitrarily defined as a systolic pressure over $150 \mathrm{mms} \mathrm{Hg}$ or a diastolic level over $90 \mathrm{mms} \mathrm{Hg}$ or both was present more frequently in the transient ischaemic attack popula-

Table 1

\begin{tabular}{|c|c|c|c|c|}
\hline$A g(y r)$ & Patic'nts & & Controls & \\
\hline $4(1-49$ & $23 \mathrm{M}$ & $16 \mathrm{~F}$ & $34 \mathrm{M}$ & $25 \mathrm{~F}$ \\
\hline $50-59$ & $41 \mathrm{M}$ & $14 \mathrm{~F}$ & $44 \mathrm{M}$ & $22 \mathrm{~F}$ \\
\hline 6()$-69$ & $43 \mathrm{M}$ & $14 \mathrm{~F}$ & $32 \mathrm{M}$ & $18 \mathrm{~F}$ \\
\hline $70-79$ & $2 \mathrm{M}$ & $2 \mathrm{~F}$ & $9 \mathrm{M}$ & $7 \mathrm{~F}$ \\
\hline Totals & $109 \mathrm{M}$ & $46 \mathrm{~F}$ & $119 \mathrm{M}$ & $72 \mathrm{~F}$ \\
\hline
\end{tabular}


tion $(55 \%$ cf $29 \%$ in males chi square $13.2 \mathrm{p}<$ $0.001 ; 44 \%$ cf $21 \%$ in females chi square $7.2 \mathrm{p}<$ $0.01)$. More male patients with transient ischaemic attacks admitted to regular smoking (77\% cf $53.5 \%$ of controls chi square $13.6 \mathrm{p}<0.001)$. The difference in smoking habit between patients with transient ischaemic attack and controls amongst the female subjects was less striking $(61 \%$ cf $47 \%$ chi square $2 \cdot 2 \mathrm{NS}$ ) (table 2).

Table 2 Blood pressure and smoking in patients with transient ischaemic attack and neurological controls:

\begin{tabular}{llll}
\hline Patient group & $\begin{array}{l}\text { BP over } 150 \mathrm{~mm} \mathrm{Hg} \\
\text { (systolic) or } 90 \\
\text { (diastolic) }\end{array}$ & Smoking \\
\hline Male & Transient Ischaemic & & \\
$\quad$ attack & $55 \%$ & $77 \%$ \\
Control & $29 \%$ & $53 \cdot 5 \%$ \\
Chi square & $13 \cdot 2$ & 13.6 \\
p value & $<0 \cdot 001$ & $<0 \cdot 001$ \\
Female Transient ischaemic & $44 \%$ & $61 \%$ \\
$\quad$ attack & $21 \%$ & $47 \%$ \\
Control & $7 \cdot 2$ & $2 \cdot 2$ \\
Chi square & $<0 \cdot 01$ & $\mathrm{NS}$ \\
\hline p-value & & \\
\hline
\end{tabular}

In view of the interrelationship between smoking, hypertension and an elevated haematocrit level an analysis of regression was performed within each sex. Age was represented by separate linear regressions in the 10 year age groups and blood pressure by linear regressions on both systolic and diastolic pressures. There was no evidence of an effect of age on haematocrit. The sex of the subject, smoking habit, and both systolic and diastolic pressure was significantly associated with haematocrit. The effect of smoking was to increase haematocrit by $1.19 \pm$ 0.59 in males and $2.18 \pm 0.68$ in females (the difference between the sexes in this effect could well be due to chance). When allowance was made for the effects of smoking and blood pressure, there was evidence that the haematocrit was higher in transient ischaemic attack patients. The estimated elevation was of $1.44 \pm 0.56$ for males and $0.75 \pm 0.75$ for females $(p<0.02)$. The difference between the male and female estimates is not significant $(\mathrm{p}=$ $0 \cdot 7)$.

\section{Discussion}

This study reaffirms the association of hypertension with early manifestations of cerebrovascular disease, obvious in the Framingham Study, ${ }^{1}$ and in the natural history data from Rochester. ${ }^{3}$ Hypertension may of itself be associated with an elevated haematocrit, ${ }^{4}$ and this trend is confirmed in our data. Similarly the smoking habit of the individual has a clear effect on haematocrit. ${ }^{2}$ Our data suggest that a smoker will have a haematocrit one to two points higher than a non-smoker, all other things being equal. In view of these associations it has been difficult to be sure whether a high haematocrit was an independent risk factor in cerebrovascular disease. The Framingham data suggested that there might be little association once the effects of hypertension etc were taken into account. Our specific study of this point reveals a small but significant independent association of the height of the haematocrit with the occurrence of transient ischaemic attacks.

A recent study 5 has suggested that a high haematocrit may be associated with the risk of carotid thrombosis and the size of any consequent cerebral infarct. There is also evidence that more platelet deposition on the wall of a vessel probably occurs in the vortex produced by a stenosis, if the haematocrit is elevated ${ }^{6}$.

It would seem prudent therefore to check the haematocrit of all patients with early manifestations of cerebrovascular disease and to re-check it after stopping smoking, treating hypertension, and possibly stopping diuretic therapy. It is questionable whether diuretics should be used to control high blood pressure in patients whose haematocrit exceeds $46 \%$ and if they are used haematocrit should be monitored closely. After these preliminary measures patients with haematocrit consistently over $52 \%$ should be investigated for polycythaemia. Whether reducing haematocrit from the high normal range (46 to $52 \%$ ) by serial venesection helps to reduce the risk of stroke, remains to be demonstrated.

We are grateful to Dr B Newman for help with statistical analysis.

\section{References}

${ }^{1}$ Kannell WB. Current status of epidemiology of brain infarction associated with occlusive arterial disease. Stroke 1971;2:295-318.

${ }^{2}$ Smith JR, Landaw SA. Smokers polycythaemia. New Engl J Med 1978;298:6-10.

${ }^{3}$ Whisnant JP, Matsumoto N, Elveback LR. Transient cerebral ischemic attacks in a community: Rochester Minnesota 1955 through 1969. Mayo Clinic Proc 1973;48:194-8.

${ }^{4}$ Letcher RL, Chien S, Pickering TG, Sealey JE, Laragh JH. Direct relationship between blood pressure and blood viscosity in normal and hypertensive subjects. Role of fibrinogen and concentration. Am J Med 1981;70:1195-202.

${ }^{5}$ Harrison MJG, Pollock S, Kendall BE, Marshall J. Effect of haematocrit on carotid stenosis and cerebral infarction. Lancet 1981;2:114-5.

${ }^{6}$ Karino T, Goldsmith HL. Adhesion of human platelets to collagen on the walls distal to a tubular expansion. Microvasc Res 1979;17:238-62. 